\title{
EFFECT OF DIFFERENT SURFACE PRETREATMENT PROTOCOLS ON THE MICRO-SHEAR BOND STRENGTH OF RESIN COMPOSITE TO RESIN INFILTRATED DEMINERALIZED ENAMEL
}

\author{
Hend S. Ahmed*, Nermeen Kamal Hamza** and Hossam A. Alhussiny ${ }^{* * *}$
}

\begin{abstract}
Aim: to assess micro-shear bond strength ( $\mu$-SBS) of composite using universal adhesive to resin infiltrated demineralized enamel after different surface pretreatments.

Methodolgy: Thirty-six intact maxillary central incisors were used. Teeth were mounted in acrylic blocks. Specimens were divided into three groups ;Group 1: teeth with sound enamel were bonded to resin composite (positive control). Group 2: teeth were demineralized then bonded to resin composite (negative control). Group 3: teeth were demineralized followed by application of (Icon-DMG). The third group was divided into four subgroups according to surface pretreatment either no surface pretreatment, abrasive roughening, air abrasion, combination of roughening and air abrasion. All specimens except positive control were demineralized by demineralizing solution. In resin infiltration group, Icon (DMG) was applied following manufacturer's instructions then specimen's surfaces were treated as mentioned before. Etch and rinse approach using universal adhesive was applied before composite packing. Five 2millimeters long micro-tubes were mounted and held in place on the uncured adhesive. The adhesive coat was then light cured and composite was packed in the microtubes. Microtubes were peeled obtaining five microcylinders for $\mu$-SBS testing. $\mu$-SBS testing was done using a universal testing machine with orthodontic wire to produce shear forces.
\end{abstract}

Results: statistically significant difference in $\mu \mathrm{SBS}$ values between different study groups was found. The highest $\mu$ SBS mean values were in positive control, grinding and air abrasion groups, followed by no treatment group, combination group. While negative control yielded lowest $\mu \mathrm{SBS}$ values.

Conclusion: surface pretreatment is mandatory to improve bonding to resin infiltrated enamel.

KEYWORDS: Demineralized enamel, white spot lesions, resin infiltration, universal adhesive and micro-shear bond strength

\footnotetext{
* Lecturer of Restorative Dentistry, Faculty of Dentistry, The British University in Egypt, Cairo, Egypt

** Associate Professor of Conservative Dentistry, Faculty of Dentistry, October University for Modern Science and Art.

*** Associate Professor of Operative Dentistry, Faculty of Dentistry, Suez Canal University, Egypt
} 


\section{INTRODUCTION}

Nowadays esthetics became one of the main patients concerns, in which one of the most common causes for the patient unacceptable esthetics is the white spot lesions on the enamel surface, especially when it affects the anterior teeth. White spot lesions appear as chalky white and opaque which is due to the subsurface enamel demineralization which is mostly located on smooth surfaces. These white spot lesions (WSL) may be due to pre- and posteruptive disorders, as fluorosis, hypoplasia, hypomineralization or molar-incisor hypo-mineralization (MIH), also one of the most common causes is dental caries, in which the first sign of enamel caries is the white spot lesions. ${ }^{14,17,18}$

Demineralized enamel shows loss of minerals within the bulk of the enamel as well as enlargement in the inter-crystalline spaces, with marked reduction in the microhardness of the sub-surface of enamel, while the external (pseudo) enamel surface remains intact which is highly mineralized comparatively with the sub-surface. The pores created within the enamel is responsible for the opaque appearance due to the difference of the refractive index between the hydroxyapatite in sound enamel and the saliva in the enamel porosities created in the WSL which will affect the scattering of light that makes the lesion appears slight opaque, meanwhile, in dried teeth saliva is replaced by air which has a greater difference in the refractive index from that of the hydroxyapatite, so lesions appear opaquer in color. Furthermore, the enamel porosities creating a pathway for the acid and the dissolved minerals decreasing the micro-hardness of enamel subsurface. . $^{2,5,8,12}$

Correct diagnosis according to the lesion depth and its activity as well as, the etiology of the lesion which is taken from the patient history is very crucial in the decision of the treatment plan and in the success of the case. The main goal of management of tooth discoloration is to produce an acceptable cosmetic result enhancing the patients smile as conservatively as possible, as well as, reinforcing the enamel mechanically and making it more acid resistant. Remineralization of the enamel can take place in the presence of saliva if there is appropriate plaque and diet control. Nowadays a great attention has been assigned to the non-invasive treatment of the enamel WSL as remineralization of the lesion using fluoride or amorphous calcium phosphatecasein phospho-peptide (ACP-CPP) which has a key role in remineralization, but this technique is not always successful as it needs great discipline and cooperation from the patient, where most patients stop the treatment protocol before its completion..$^{10,14}$

Therefore, an alternative approach using the resin infiltration technique which is a micro-invasive technique, by sealing the enamel pores with low viscosity resin Tri-ethylene glycol di-methacrylate (TEGDMA) creating a diffusion barrier within the lesion, preventing acids from penetration creating an acid resistant layer as well as, increasing the hardness of enamel by reinforcing the enamel crystallites and improving the esthetics as it has refractive index almost similar to that of the hydroxyapatite of sound enamel. In some cases, the resin infiltration technique does not give the camouflage effect which may be due to the incomplete resin infiltration when the pores are too deep, therefore, in some of the cases it needs to be masked by resin composite to reach the esthetic goal. ${ }^{13,14}$

Multimode or universal adhesives are commonly used for simplification of the adhesive technique and for improving the clinical versatility, which consists of one bottle adhesive that can be used in etch and rinse or self-etch strategies. Combining the acidic functional monomer 10 MDP with Vitrebond Copolymer both compounds interact with the calcium from the hydroxyapatite, forming the $\mathrm{Ca}$ 10-MDP which is hydrolytically stable. ${ }^{11}$

Therefore, the aim of the present study was to assess the micro-shear bond strength of the resin composite using universal adhesive system in the etch and rinse mode to resin infiltrated 
demineralized enamel after different surface pretreatment protocols. The null hypothesis was that the different surface pretreatment protocols have no effect on bond strength of composite to resin infiltrated demineralized enamel.

\section{MATERIALS AND METHODS}

\section{Sample size calculation}

Sample size calculation was conducted using G*Power 3.1.9.4 Software based on data obtained from a previous study (Borges et al, 2019). The power of t-test was set at $80 \%$ using a two-tailed significance level of 5\%. A sample size of 6 human incisors per group was estimated, for a total of 36 incisors per 6 groups.

\section{Teeth selection}

Thirty-six extracted intact maxillary central incisors extracted for periodontal reasons were selected and stored in a thymol solution $(0.025 \%)$ until the day of use. All teeth were examined under a stereo microscope to exclude teeth with cracks, restorations or developmental defects. Crowns were sectioned parallel to the incisal plane $1 \mathrm{~mm}$ below the cementoenamel junction (CEJ) using a watercooled slow-speed diamond saw (IsoMet 5000 Precision Saw; Buehler, Lake Bluff, Illinois). The teeth were cleaned with brush and pumice-water suspension.

\section{Sample fixation}

Each crown was embedded in self-cured acrylic resin (Acrostone, Cairo, Egypt) square block of (1.5 $\mathrm{cm} X 1.5 \mathrm{~cm}$ X 2cm). The blocks were prepared using split mold assembly that allow for pouring of five blocks at the same time. Each tooth crown was embedded in acrylic resin while it was in soft dough stage and the crown was pressed in the acrylic so that the labial surface faced upwards till it was flushed with the top surface of the block and the lingual surface faced the acrylic resin. The embedded samples were allowed to polymerize at room temperature. After acrylic setting the block was removed from the mold and checked carefully.

\section{Specimens grouping}

Specimens were divided into three main groups as follows; Group $1(n=6)$ : teeth with sound enamel were bonded to resin composite to act as positive

TABLE (1): Name and product details of the materials used in this study

\begin{tabular}{|c|c|c|c|c|}
\hline Material & Description & Composition & Manufacturer & \\
\hline $\begin{array}{l}\text { Single Bond } \\
\text { Universal }\end{array}$ & $\begin{array}{l}\text { Universal } \\
\text { adhesive }\end{array}$ & $\begin{array}{l}10 \text { MDP Phosphate Monomer, Dimethacrylate } \\
\text { resins, HEMA, Vitrebond TM Copolymer, filler, } \\
\text { Ethanol, Water, Initiator and Silane }\end{array}$ & $\begin{array}{l}\text { 3M ESPE } \\
\text { Dental products, } \\
\text { St.paul,MN, USA }\end{array}$ & 5210184 \\
\hline Z350 XT & $\begin{array}{l}\text { Nanofilled } \\
\text { resin } \\
\text { composite }\end{array}$ & $\begin{array}{l}\text { Fillers:Non-agglomerated } 20 \mathrm{~nm} \text { silica filler, } \\
\text { Non-agglomerated } 4 \text { to } 12 \mathrm{~nm} \text { Zirconia filler, } \\
\text { Aggregated Zirconia/silica cluster. Filler } \\
\text { loading } 55.6 \% \text { by volume and } 72.5 \% \text { by } \\
\text { weight. Matrix:BISGMA,UDMA,TEGDMA } \\
\text { PEGDMA and BIS-EMA. }\end{array}$ & $\begin{array}{c}\text { 3M ESPE } \\
\text { Dental products, } \\
\text { St.paul,MN, USA }\end{array}$ & N952379 \\
\hline Icon (DMG) & $\begin{array}{l}\text { Resin } \\
\text { infiltration } \\
\text { system }\end{array}$ & $\begin{array}{l}\text { Icon-Etch (DMG) : 15\% hydrochloric acid, } \\
\text { water, pyrogenic silica, surfactant, pigments. } \\
\text { Icon-Dry (DMG) : Ethanol. Icon-Infiltrant } \\
\text { (DMG): TEGDMA-based resin, initiators and } \\
\text { stabilizers }\end{array}$ & $\begin{array}{c}\text { DMG, Hamburg, } \\
\text { Germany }\end{array}$ & 626384 \\
\hline
\end{tabular}


control. Group $2(n=6)$ : teeth were subjected to demineralization (artificial caries) to simulate white spot lesions then bonded to resin composite to act as negative control. Group $3(n=24)$ : teeth were subjected to demineralization (artificial caries) to simulate white spot lesions and followed by application of resin infiltration (Icon-DMG). The third group was divided into four subgroups (6 each) according to the surface pretreatment before bonding to composite either no surface pretreatment, roughening by abrasive alone, air abrasion alone, combination of roughening and air abrasion.

\section{Demineralization of enamel}

All the specimens except the positive control specimens were demineralized by suspending all the teeth into jar containing $200 \mathrm{ml}$ of demineralizing solution $\left(\mathrm{CaCl}_{2}=2.2 \mathrm{mM}, \mathrm{Na} \mathrm{H}_{2} \quad \mathrm{PO}_{4}=2.2 \mathrm{mM}\right.$, Lactic acid $=0.05 \mathrm{M}$, Fluoride $=0.2 \mathrm{ppm}$, solution was adjusted with $50 \% \mathrm{Na} \mathrm{OH}$ to $4.5 \mathrm{pH}$ ). The specimens were left for 72 hours, in an incubator at a temperature of $37^{\circ} \mathrm{C}$.

\section{Resin infiltration}

In the resin infiltration group, the resin infiltration system Icon (DMG) was used. The infiltration procedure was carried out in according to the manufacturer's instructions: (1) Icon-Etch (15\% hydrochloric acid) was applied for two minutes and then the specimens were rinsed with water and air dried for 30 seconds; (2) Icon-Dry (ethanol) was applied for 30 seconds and air dried; (3) Icon-Infiltrant was applied two times, the first time for three minute and the second time for one minute; (4) both applications were light cured for 40 seconds using a LED light-curing unit (Elipar S10, 3M ESPE, St Paul, MN, USA) operating in standard mode at light intensity $1200 \mathrm{~mW} / \mathrm{cm}^{2}$ according to manufacturer instructions.; (5) specimens were polished with aluminum oxide abrasive papers (4000 grit; FEPA-P, Extec) for 20 seconds, to remove the surplus material.

\section{Enamel surface preparation before bonding}

Subgroup 1: no surface treatment and the resin infiltrated enamel surface was left intact and the universal adhesive was applied with etch and rinse mode according to manufacturer's instructions.

Subgroup 2: grinding with diamond stone, the labial surface was lightly ground using water-cooled diamond no. 848 medium (Brassler, Savannah, Georgia, USA) and the universal adhesive was applied with etch and rinse mode according to manufacturer's instructions.

Subgroup 3: air abrasion; the air abrasion system used in this study was (AquaCare- Velopex International) that was adjusted at 60 psi particle energy, $53 \mu \mathrm{m}$ mean aluminum oxide particle size, 5 $\mathrm{g} / \mathrm{min}$ jet intensity, 20-s application, $10 \mathrm{~mm}$ distance and 90-degree impingement angle. The particle reservoir was frequently agitated to homogenize the size distribution. The universal adhesive was applied with etch and rinse mode according to manufacturer's instructions.

Subgroup 4: combination of grinding followed by air abrasion and the universal adhesive was applied with etch and rinse mode according to manufacturer's instructions.

\section{Microshear bond strength testing}

\section{Bonding procedure}

Etch and rinse approach using the universal adhesive Single bond universal (3M ESPE, St Paul, MN, USA) was applied according to manufacturer's instructions before resin composite packing. All enamel specimens were etched with phosphoric acid etchant for 30 seconds then rinsed with water for another 30 seconds and finally airdried for 10 seconds leaving the specimens with the chalky white appearance. Two consecutive coats of the adhesive were applied using disposable micro-brushes (Micro-brush-International, Grafton, USA). The first coat was applied with agitation for 
20 seconds and left undisturbed for 5 seconds, and then air thinned till no movement of the adhesive is observed then the second coat was applied with the same technique. The adhesive was left uncured to facilitate fixation of the cut microtubes on it before packing of the resin composite into them. The disposable Micro-brushes were replaced after each use.

\section{Resin composite packing for micro-shear bond strength}

Two millimeters long polyethylene microtubes were cut from Tygon ${ }^{\circledR}$ tube (R-3603, Norton Performance Plastic Co., Cleveland, USA) with an internal diameter of $0.8 \mathrm{~mm}$ measured using RadioShack Electronic Digital Caliper for standardization. Five micro-tubes were mounted and held in place with a tweezer on the uncured adhesive to restrict the bonding area.The adhesive coat was then light cured for 10 seconds using a LED light-curing unit (Elipar S10, 3M ESPE, St Paul, MN, USA) operating in standard mode at light intensity $1200 \mathrm{~mW} / \mathrm{cm}^{2}$ according to manufacturer instructions. During light-curing procedure, the light cure tip was held on the top of the mounted micro-cylinder to standardize the distance of curing ( $2 \mathrm{~mm}$ from the adhesive). After curing of the adhesive, nano-filled resin composite (Z350 XT, 3M ESPE, St Paul, MN, USA) was packed in the micro-cylinders using endodontic plugger. The packed resin composite in the cylinders was light-cured for 20 seconds using the same LED light curing unit with a plastic matrix strip placed over the resin composite. Using a sharp scalpel and after one hour of storage at room temperature the polyethylene micro-tubes were peeled away by making two opposite cuts using the tip of scalpel blade number 11 in the micro tube, obtaining composite micro-cylinders of $0.8 \mathrm{~mm}$ diameter and $2 \mathrm{~mm}$ height bonded to the specimen surface. The specimens were stored in distilled water for 24 hours prior to micro shear bond strength $(\mu$-SBS) testing. $\mu$-SBS testing was done using a universal testing machine (Lloyd Instruments Ltd., Fareham UK). A wire of 0.2-mm diameter was looped around the resin composite micro-cylinder, making contact through half its circumference, and was gently held flush against the resin-tooth interface. The resintooth interface, the wire loop and the center of the load-cell were aligned as straight as possible to ensure the correct application of the shear force. A shearing load with tensile mode of force was applied via materials testing machine at a crosshead speed of $0.5 \mathrm{~mm} / \mathrm{min}$. The relatively slow crosshead speed was selected in order to produce a shearing force that resulted in debonding of the micro cylinder along the substrate-adhesive interface. The load required to debonding was recorded in Newton.

Micro-Shear bond strength calculation:

- The load at failure was divided by bonding area to express the bond strength in MPa :

$\tau=\mathrm{P} / \pi \mathrm{r}^{2}$

Where; $\tau=$ micro-shear bond strength (in $\mathrm{MPa}$ ).

$\mathrm{P}=$ load at failure (in N). $\pi=3.14$

$\mathrm{r}=$ radius of micro-cylinder (in $\mathrm{mm}$ ) which was checked with a digital caliper before the test.

\section{Statistical Analysis}

Statistical analysis was performed using IBM SPSS Statistics Version 20. Data was presented as mean and standard deviation (SD). Significance level was set at $\mathrm{P}=0.05$. Kolmogorov-Smirnov and Shapiro-Wilk tests were used to assess data normality. One-Way ANOVA and Tukey's HSD post-hoc test were conducted to compare $\mu \mathrm{SBS}$ values between different study groups.

\section{RESULTS}

One-Way ANOVA followed by Tukey's HSD post-hoc test (Table 2) showed that there was a statistically significant difference in $\mu$ SBS values 
between different study groups $(\mathrm{P}<0.001)$. The significantly highest $\mu \mathrm{SBS}$ mean values were recorded in positive control, ICON + grinding and ICON + air abrasion groups, which did not differ significantly. This was followed by the mean value of ICON + no treatment group, which was significantly higher than that of ICON + combination group. While the negative control yielded the significantly lowest $\mu$ SBS values among the other study groups.

TABLE (2) Mean \pm SD for the effect of different surface treatments on $\mu \mathrm{SBS}(\mathrm{MPa})$ to demineralized enamel.

\begin{tabular}{|c|c|}
\hline & $\mu \mathrm{SBS}$ \\
\hline Positive control & $29.28 \pm 0.98^{\mathrm{a}}$ \\
\hline Negative control & $14.28 \pm 2.09^{\mathrm{e}}$ \\
\hline ICON + No treatment & $22.72 \pm 1.42^{\mathrm{c}}$ \\
\hline ICON + Grinding & $27.93 \pm 1.04^{\mathrm{a}}$ \\
\hline ICON + Air abrasion & $27.65 \pm 1.10^{\mathrm{a}}$ \\
\hline ICON + Combination & $19.69 \pm 1.81^{\mathrm{d}}$ \\
\hline P-value & $<0.001^{*}$ \\
\hline
\end{tabular}

*: significant at $P \leq 0.05 ;$ NS: non-significant at $P>0.05$

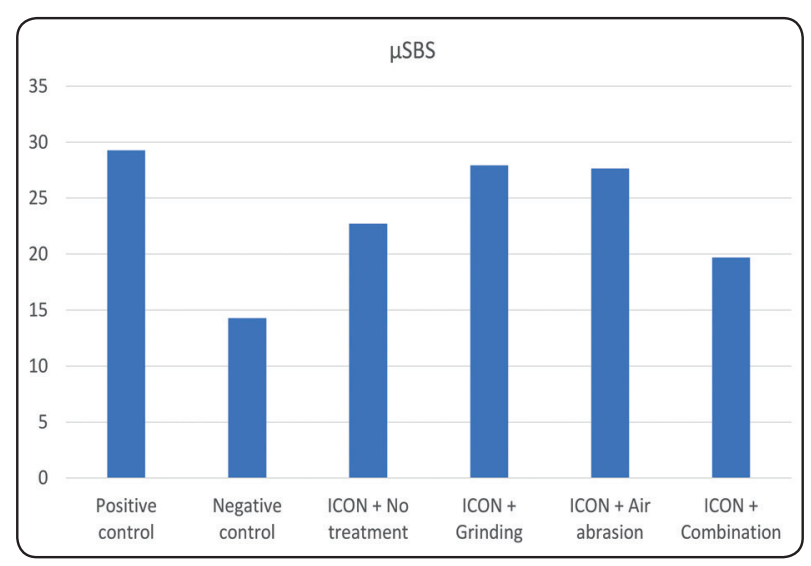

Fig. (1): Histogram showing the mean $\mu$ SBS of resin composite after different surface treatments

\section{DISCUSSION}

The more understanding of dental caries, as well as, its early diagnosis, gave the chance for modern dentistry to shift from the invasive approach to the prevention, non-invasive and minimal invasive approaches. White spot lesions are one of the early signs of dental caries on the enamel surface, in which the hydroxyapatite has been exposed to demineralization in the enamel sub-surface leaving an apparently intact or pseudo intact surface that covers the mineral deprived area with decreased enamel hardness. ${ }^{10,12}$

Resin infiltration technique is one of the noninvasive approaches used to manage the white spot lesions, which depends on the occlusion of the pores within the body of the caries lesion. It has been widely used in the past years as a method of arresting the caries, increasing the hardness of enamel and improving the esthetics by displacing the water or air present within the porosities found in the enamel sub-surface. ${ }^{10,13}$ Several studies were made in order to improve the penetration of the infiltrant resin in the carious lesions, but it is still questioned whether its application on demineralized enamel can be applied prior the application of resin composite? Therefore, the aim of the present study was to evaluate the micro-shear bond strength of resin composite using universal adhesive on resin infiltrated demineralized enamel with different surface pretreatment.

Artificial carious lesion was created in order to simulate in-vivo caries development, which is produced with similar characteristics as found in natural lesions. The demineralized specimens were treated with a resin infiltration (Icon Infiltrant) using $15 \% \mathrm{HCl}$ which allows better resin penetration by removing the mineral layer and opening wider surface porosities to boost the resin infiltration of the more porous sub-surface body of the lesion, then $99 \%$ of ethanol was applied to eliminate water that is stored inside the micro-porosities of the body of 
the lesion which will allow better penetration of the resin by capillary force..$^{5}$ Multimode or universal adhesive system was used in this study with the etch and rinse mode, as etching of enamel allows selective demineralization of the hydroxyapatite crystals, which increase the surface roughness and the surface energy which allows better wettability capacity of the resinous monomers which when polymerized allows the formation of resin tags that anchor the resin to the enamel. ${ }^{5,6,18}$

The results of the present study showed that surface pretreatment of the resin infiltrated demineralized enamel had a great effect on the micro-shear bond strength of the resin composite, in which the resin infiltrated enamel group with no surface pretreatment showed higher bond strength than that of the untreated demineralized enamel. Accordingly, the null hypothesis was rejected. The results were inconvenience with Marina, S. et al, ${ }^{12}$ meanwhile on comparing the abovementioned group with untreated sound enamel group there was a significant difference in the micro-shear bond strength. Attin et al ${ }^{3}$ who demonstrated that the shear bond strength was significantly reduced with the demineralized enamel that had been pretreated with resin infiltrant on comparison with sound enamel and these results were in connivence with the results of the present study. These results were in contradiction with Alessandra, B. et al ${ }^{2}$ who demonstrated that the infiltrated enamel showed similar bond strength as sound enamel and this was explained that its due to the affinity between the monomers present in the infiltrant and the monomers of the adhesive system, clarifying the thick oxygen inhibited layer formed within the infiltrant. Using aluminum oxide abrasive paper as per manufacturer instruction of the resin infiltration technique to remove the excess resin from the surface diminish the oxygen inhibited layer formed which may negatively affect the chemical connection between the resin infiltrant to the monomers of the adhesive system.
Regarding the surface pretreatment of the resin infiltrated demineralized enamel, according to Yim et al ${ }^{19}$ the surface preparation method of enamel significantly affects the bond strength of the adhesives. In morphological analysis of the enamel surface Dotty et al ${ }^{7}$ reported that using air abrasion for surface enamel treatment presents an irregular pattern with medium depth of $4.15 \mathrm{~mm}$, therefore association of the air abrasion with the acid etching gave the highest bond strength according to Canay et al. ${ }^{4}$ As well as, the use of diamond bur for the enamel surface pretreatment increases the surface area, which subsequently increase the mechanical retention according to Abd ElKadir Sengun et al ${ }^{1}$. In the present study using diamond stone or air abrasion techniques significantly improved the micro-shear bond strength of resin composite in which there was no significant difference with the sound enamel group, this may be due to the occlusion of the micro-porosities present in the body of the lesion by resin infiltrant which increases the acid resistance of the enamel surface, as it is investigated in many researches its great affinity in arresting caries lesions making enamel more acid resistant. By using the universal adhesive in the etch and rinse mode the enamel surface becomes more resistant to the acid etch as well as the 10 MDP and Vitrebond copolymer present in the universal adhesive that allows the chemical bond with the mineral content in enamel is hindered, as the demineralized enamel is subjected to $15 \% \mathrm{HCl}$ to promote erosion of the surface layer which removes more minerals from the enamel surface. ${ }^{5}$ Therefore, by using air abrasion or diamond stone as surface pretreatment before application of the adhesive system, increases the surface roughness creating irregularities in the enamel surface, which increase the micromechanical retentive areas increasing the bond strength ${ }^{20}$.

The prepared enamel surface does not posses high surface energy due to the presence of smear layer, acid etching allows the removal of the smear layer created by air abrasion or the diamond bur to 
obtain good bond, as the smear layer will prevent the diffusion of the monomers into the superficial dental structure. ${ }^{9,15,16}$. Therefore, on using combined techniques (air abrasion and diamond stone) of the resin infiltrated demineralized enamel, the bond strength was significantly decreased when compared with the sound enamel, but still higher than that of the demineralized enamel surface. This decrease in bond strength may be due to the thickness of smear layer formed on the enamel surface that acid etching was not able to remove completely that affected the bond strength negatively by limiting the infiltration of the adhesive to the maximum depth of the formed surface irregularities which are filled with the smear layer.

Based on the results of the present study, it was concluded that when restorative treatment is indicated with resin infiltrated demineralized enamel, surface pretreatment is mandatory to improve bonding to it. Moreover, the method of surface pretreatment is very crucial as it will affect the bond strength of the final restoration. Grinding and air abrasion are found to be the best pretreatment protocols with the resin infiltrated enamel before bonding with etch and rinse mode of the universal adhesive.

\section{REFERENCES}

1. Abdulkadir Sengun, Hasan Orucoglu, Ilknur Ipekdal and Fusun Ozer (2008), Adhesion of Two Bonding Systems to Air-Abraded or Bur-Abraded Human Enamel Surfaces. Eur. J. Dent., 2, pp. 167-175.

2. Alessandra Buhler Borges, Amjad Abu Hasna, Amanda Guedes Noguiera Matuda, Stephanie Ribeira Lopes, Ana Paula Valenta, Pinho Mafetano, Aline arantes, Angelica Ferreira Duarte, Daphne Camara Barcellos, Carlos Rocha Gomes Torres and Cesar Rogerio Pucci (2019), Adhesive systems effect over bond strength of resin infiltrated and de/remineralized enamel, F1000Research, 8:1743.

3. Attin R., Stawarcyzk B., Kecik D., Kodel M., wiechman D. and Attin T. (2012), Shear bond strength of brackets to demineralized enamel after different pretreatment methods. Angles Ortho., 22, pp. 56-61.
4. Canay S, Kocaderelli I,Akça E. (2000) The effect of enamel air abrasion on the retention of bonded metallic orthodontic brackets. Am J Orthod Dentofacial Orthop.,117, pp. 15-19.

5. Carlos Rocha Gomes Torres, Alessandra Buhler Borges, Luciana Silva Gomes and Rodrigo Simoes de Oliveira (2011), Effect of caries infiltration technique and fluoride therapy on the colour masking of white spot lesions. Journal of Dentistry, 39, pp. 202-207.

6. Curry J. A. and Tenuta L. M. (2009), Enamel remineralization: controlling the caries disease or treating early caries lesions? Braz. Oral Res., 23 (Supp 1), pp. 23-30.

7. Dotty WD, Pettey D, Holder R, Philips S. KCP (1994), Enamel etching abilities tested. J Dent Res., 73,pp. 411

8. Elif Beril Gurdogan, Didem Ozdemir-Ozenen and Nuket Sandalli (2017), Evaluation of Surface Roughness Characteristics Using Atomic Force Microscopy and Inspection of Microhardness Following Resin Infiltration with Icon. Journal of Esthetic and Restorative Dentistry , 29 (3), pp. 201-208

9. Jahn KR, Geitel B, Kostka E, Wischnewski R, Roulet JF. (1999), Tensile bond strength of composite to air-abraded enamel. J Adhes Dent., 1, pp. 25-30

10. Jorge Perdigao (2019), Resin infiltration of enamel white spot lesions: An ultramorphological analysis, Journal of Esthet. Restor. Dent., 32 (3), pp. 317-324.

11. Mario Fernando de Goes, Mirela Sanae Shinohara and Marcela Santiago Freitas (2014), Performance of a new one step multi-mode adhesive on etched and non-etched enamel on bond strength and interfacial morphology, J. Adhes. Dent., 16, pp. 243-250.

12. Marina Simanovic Anicic, Cecillia Goracci, jelena Juloski, Ivana miletic and Senka Mestrovic (2020), The influence of resin infiltration pretreatment on orthodontic bonding to demineralized human enamel, Journal of Applied Science, 10,3619 .

13. Nadia Malek Taher, Haifa Abdulrahman Alkhamis and Sarah Mesha'l Dowaidi, (2012), The influence of resin infiltration system on enamel microhardness and surface roughness: An in vitro study. Saudi Dental journal, 24, pp. 79-84.

14. Novjots S. Mann, Sameer Makkar and Prana V. Gupta (2015), Resin infiltration technique: The minimal invasive approach to treat with spot lesions, TMU. J. Dent., 2 (2), pp. 71-74. 
15. Rinaudo PJ, Cochran MA, Moore BK. (1997), The effect of air abrasion on shear bond strength to dentin with dental adhesives. Oper Dent., 22, pp. 254-259.

16. Roeder LB, Berry EA, 3rd, You C, Powers JM. (1995), Bond strength of composite to air-abraded enamel and dentin. Oper Dent., 20, pp. 186-190.

17. Sophie Doméjean, Raphaël Ducamp Stéphanie Léger and Christopher Holmgren (2015), Resin Infiltration of NonCavitated Caries Lesions: A Systematic Review. Med princ pract., 24(3), pp. 216-221

18. Torres CRG., Rosa PCF. and Ferreira N. S. (2012), Effect of caries infiltration technique and fluoride therapy on microhardness of enamel carious lesions., Journal of Oper. Dent., 37 (4), pp. 363-369.

19. Hyun-Kyung Yim, Ji-Hyun Min, Ho-Keun Kwon, Baek-Il Kim (2014), Modification of surface pretreatment of white spot lesions to improve the safety and efficacy of resin. infiltration. Korean J Orthod., 44(4), pp. 195-202.

20. Tulga F, Kara D. (1998), The evaluation of the effects of various surface treatments and acid-etching times on tensile bond strengths of fissure sealants on primary teeth (Part II) GU Dishekimligi Fakultesi.15, pp. 41-50. 
